\title{
Evaluation of the Safe Ischemic Time of Clamping During Intermittent Pringles Maneuver in Rabbits
}

\author{
Mohsen Kolahdoozan,', Akbar Behdad," Mehrdad Hosseinpour," Samin Behdad, ${ }^{2, *}$ and \\ Mohammad Taghi Rezaei ${ }^{1}$ \\ ${ }_{1}^{1}$ Department of General Surgery, Isfahan University of Medical Sciences, Isfahan, IR Iran \\ ${ }^{2}$ Trauma Research Center, Kashan University of Medical Sciences, Kashan, IR Iran \\ ${ }^{*}$ Corresponding author: Samin Behdad, Trauma Research Center, Kashan University of Medical Sciences, Kashan, IR Iran. Tel: +98-3155540026, Fax: +98-36262828, \\ E-mail: samin.behdad@yahoo.com \\ Received 2015 July 6; Revised 2015 August 31; Accepted 2015 September 10.
}

\begin{abstract}
Background: The liver is the most commonly injured organ in blunt abdominal trauma. Although major hepatic bleeding may be partially controlled with portal triade clamping (the Pringle's maneuver), continuous prolonged clamping results in liver ischemia.

Objectives: The purpose of this study was to determine the safe time of Pringle maneuver based on pathologic changes of liver in rabbit models.

Materials and Methods: In an experimental study, 20 New-Zealand white rabbits were selected. In laparotomy, a blunt dissector was passed through the foramen of Winslow and the hepato-duodenal ligament encircled with an umbilical tape. En masse Pringle maneuver was performed using atraumatic flexible clamps. Rabbits were divided into four groups based on Pringle maneuver time (30 minutes, 45 minutes, 60 minutes, and 75 minutes). A hepatic biopsy was performed at the beginning of operation. The degree of tissue injury was evaluated using blood markers.

Results: There were five rabbits in each group. At the end of 60 minutes ischemia, only minor alterations were observed in pathological specimens. At the end of 75 minutes, hepatocyte damage and necrosis were observed. The serum levels of alanine aminotransferase (Group A: $\mathrm{P}=0.02$; Group B: $\mathrm{P}=0.01$; Group $\mathrm{C}: \mathrm{P}=0.0002$; Group D: $\mathrm{P}=0.01$ ) and Aspartate aminotransferase (Group A: $\mathrm{P}=0.03$; Group B: $\mathrm{P}$ $=0.002$; Group C: $\mathrm{P}=0.0004$; Group D: $\mathrm{P}=0.0003)$ were significantly increased post-operatively. The maximum level was in the first day after operation.

Conclusions: Continuous portal triade clamping (the Pringle maneuver) during liver ischemia (30 and 45 minutes) in rabbits resulted in no ischemic change. Increasing time of clamping to 30 minutes was safe in intermittent Pringle maneuver.

Keywords: Liver, Pringle’s Maneuver, Ischemia
\end{abstract}

\section{Background}

The liver is the most commonly injured organ in blunt abdominal trauma. It is also the second most commonly injured organ in penetrating abdominal trauma $(1,2)$. The high vascularity and anterior location of liver in abdomen increase susceptivity of liver from traumatic mechanism.

The treatment strategy in a patient with blunt liver trauma is determined by the patient's hemodynamic status. In stable hemodynamic patients non-operative management such as observation and possibly arteriography and embolization controled the injury (1-4). Laparatomy is mandatory in severe hemoperitoneum due to liver injury, unstable hemodynamic patients and those who fail non-operative management (5).

The basic damage control technique for control of hepatic hemorrhage is peri-hepatic packing. This manoeuver, when performed properly, would arrest most hemorrhage except for major arterial bleeding. It is important to recognize that liver packing would not control arterial bleeding and any bleeding artery should be sutured/ligated prior to liver packing (6). Major hepatic bleeding may be partially controlled with a soft vascular clamp on the portal triad (the Pringle's maneuver). Clamping the hepatoduodenal ligament during partial liver resections results in temporary occlusion of the portal vein and hepatic artery. Continuous clamping results in one prolonged period of ischemia followed by reperfusion, whereas during intermittent clamping multiple short periods of ischemia are induced, all followed by a reperfusion episode (7).

As a consequence, ischemia and subsequent reperfusion result in a complex metabolic, (8) immunological, $(9,10)$ and microvascular (11) changes, which together might contribute to hepatocellular damage and dysfunction. Ischemia decreases the delivery of oxygen and substrates to a level inadequate to maintain cellular energy $(10,12)$.

\section{Objectives}

Considering lack of adequate information for optimal ischemia time for liver, the present study was conducted

Copyright (c) 2015, Kashan University of Medical Sciences. This is an open-access article distributed under the terms of the Creative Commons Attribution-NonCommercial 4.0 International License (http://creativecommons.org/licenses/by-nc/4.0/) which permits copy and redistribute the material just in noncommercial usages, provided the original work is properly cited. 
to determine the safe time of Pringle maneuver based on pathologic changes of liver in rabbit models.

\section{Materials and Methods}

The ethics committee for animal research of the Medical University of Isfahan approved the study. In an experimental study, 20 New-Zealand white female rabbits (1.2 \pm $0.3 \mathrm{~kg}$ ) were selected. The rabbits were housed in a control environment with an ambient room temperature of $24^{\circ} \mathrm{C}$ and artificial illumination (12 hours light-dark cycle) for at least two days before starting the experiment. They were fasted for 4 hours before the experiment, but had free access to tap water. General anesthesia was induced by intravenous injection of ketamine hydrochloride ( 35 mg per $\mathrm{Kg}$ ) in paraspinal muscles accompanied by oxygen at $0.5 \mathrm{~L} /$ minute. Hairs of surgical site was shaved and the skin was disinfected with $2 \%$ iodine tincture and positioned in supine position. The feet were fixed to the surgical table. After preparation liver was accessed through a midline incision. The hepatoduodenal ligament is usually clamped en masse with an atraumatic vessel clamp to eliminate all accessory arterial supply to the liver. Once the lesser omentum is opened, a blunt dissector may be passed through the foramen of Winslow and the hepatoduodenal ligament encircled with an umbilical tape. En masse Pringle maneuver performed using atraumatic flexible clamps.

Rabbits divided into four groups underwent a Pringle maneuver (PM): group A $(n=5)$ submitted to a continuous inflow occlusion for 30 minutes; group $B(n=5)$ underwent a PM for 45 minutes; group C ( $n=5)$ underwent a PM for 60 minutes and group D $(n=5)$ underwent a PM for 75 minutes. A hepatic biopsy was performed at the beginning of operation. The degree of tissue injury was evaluated using blood markers (aspartate aminotransferase (AST), alanine aminotransferase (ALT), one hour before laparotomy and 1- 7 days after the operation.

After ligation of hepatic pedicle, a wedge-shaped liver biopsy of approximately $0.5 \mathrm{~cm}^{3}$ was taken from the right liver lobe based on group type (Group A at 30 minutes, Group B at 45 minutes, Group C at 60 minutes, and Group $\mathrm{D}$ at 75 minutes). At the end of operation, the peritoneum and fascia were closed with continuous sutures using absorbable thread and the skin layer was closed separately using the same method. For light microscopy, the specimens were fixed in $4 \%$ buffered formaldehyde and routinely processed.

For laboratory analyzes, blood samples were taken from peripheral vein preoperation and postoperation in first, third, fifth and seventh days for comparison of serum levels of AST and ALT. All data presented as mean \pm SD. The paired $\mathrm{t}$-test was used to compare variables between the groups. Statistical analyses were performed using SPSS 11 software.P value less than 0.05 was considered as significant.

\section{Results}

The groups were similar regarding age and sex (female).
There were five rabbits in each group. The mean body weight of rabbits was $1.2 \pm 0.3 \mathrm{~kg}$.

\subsection{Liver Staining Data}

Light microscopic evaluation of liver biopsies obtained before ischemia showed normal liver. The same was observed in Group A. In Group B vascular congestion of central venule and portal venule and moderate to severe sinozoidal hemorrhage were seen (Figures 1 and 2). At the end of 60 minutes ischemia, only minor alterations were observed (Figure 3). These consisted of some parenchymal cell swelling with cytoplasmic microvacularization, predominantly in the midzonal areas. At the end of 75 minutes, hepatocyte damage and necrosis observed (Figure 4).

\subsection{Biochemical Data}

Preoperative liver function test did not differ between the groups (Table 1, Figures 5 and 6). In Figures 5 and 6 , liver function tests are compared pre- and postoperatively between the groups.

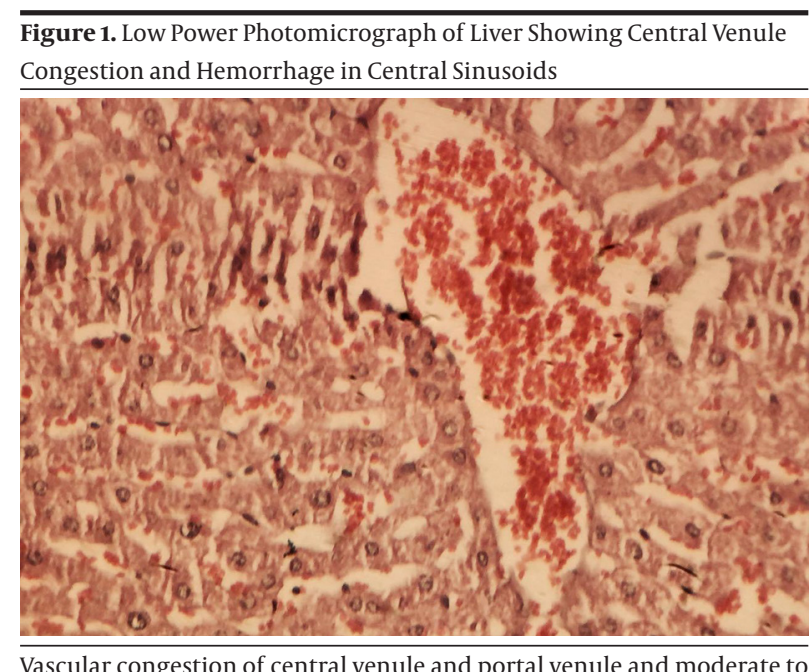
severe sinusoidal hemorrhage $(\times 100)$.

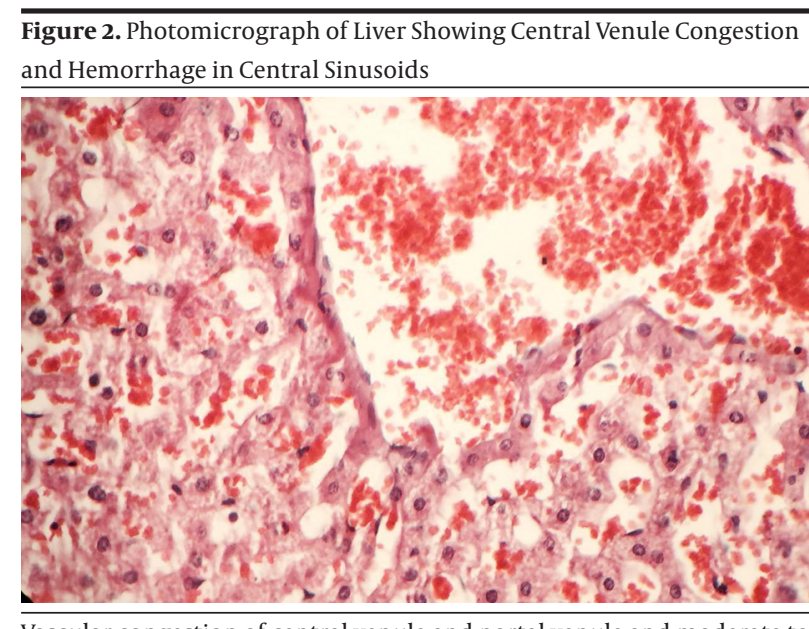

Vascular congestion of central venule and portal venule and moderate to severe hemorrhage $(\times 400)$. 
Kolahdoozan Met al.

Figure 3. Photomicrograph of Liver Showing Portal Space Inflammation Including Dominantly Lymphocytes

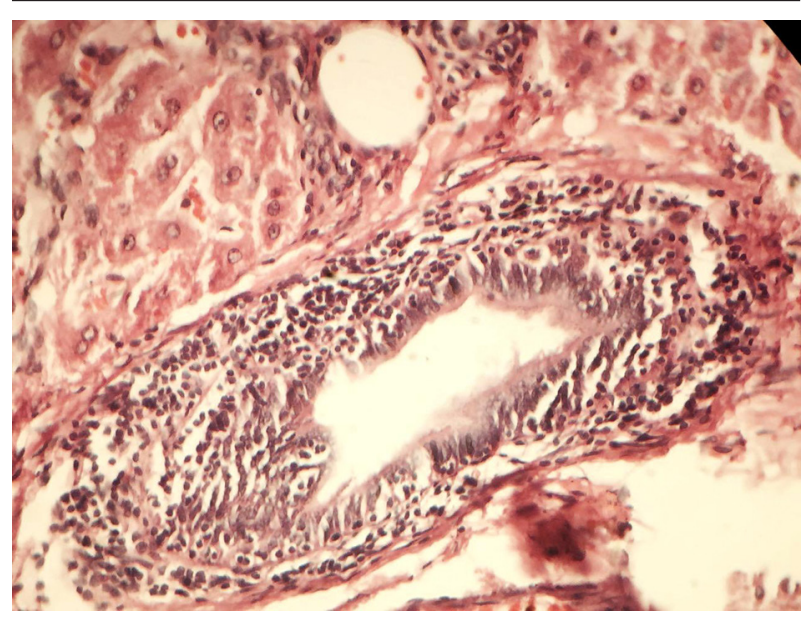

Vascular congestion of central venule and portal venule, severe hemorrhage and mixed inflammatory cells in portal spaces $(\times 400)$.
Figure 4. Photomicrograph of Liver Showing Ballooning Degeneration of Hepatocytes

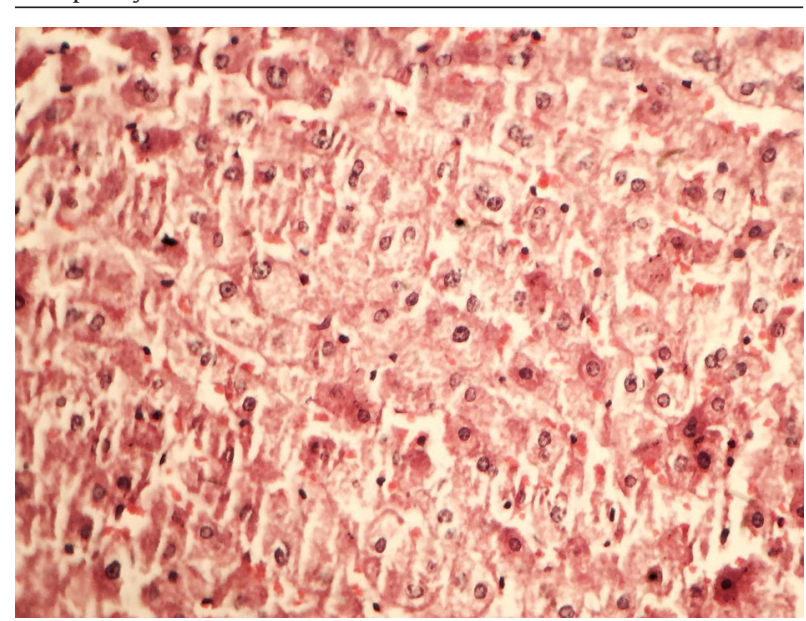

Vascular congestion of central venule and portal venule, severe hemorrhage, mixed inflammatory cells in portal spaces, and ballooning degeneration of hepatocytes $(\times 400)$.

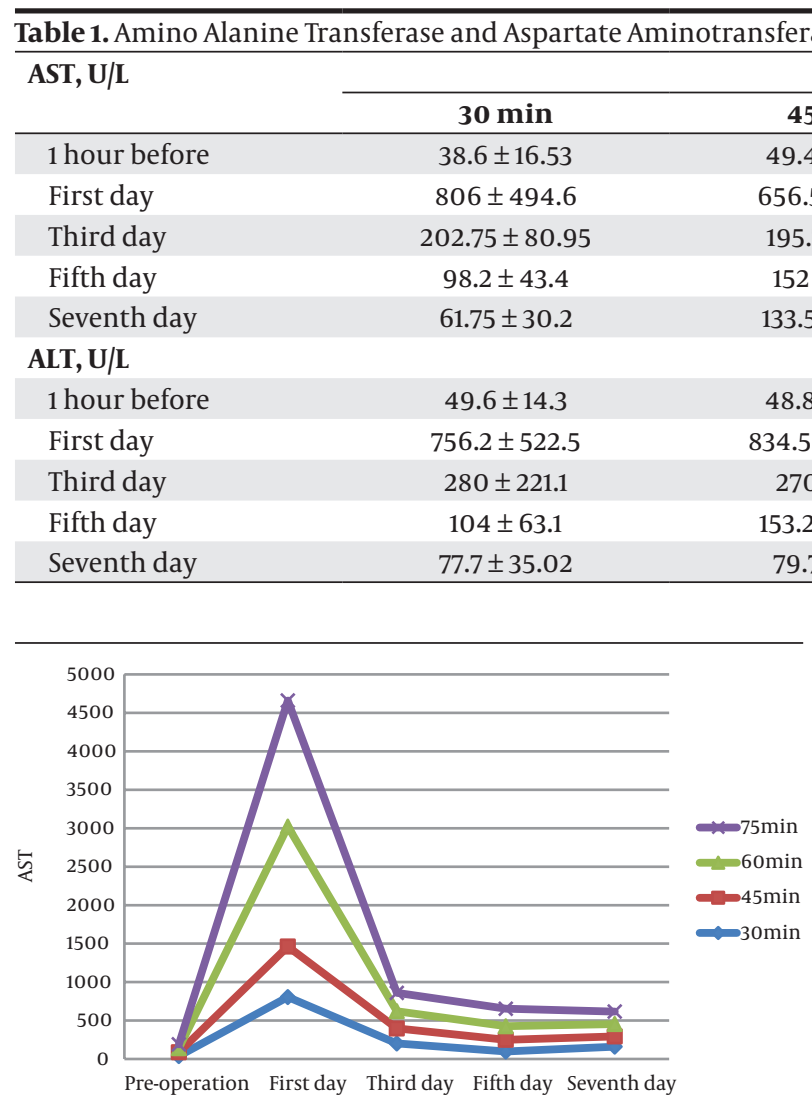

Figure 5. Aspartate Aminotransferase Serum Activities Pre-Operatively to Seventh Day Post-Operative

The serum levels of ALT (Group A: $\mathrm{P}=0.02$; Group $\mathrm{B}: \mathrm{P}=0.01$; Group C: $\mathrm{P}=0.0002$; Group $\mathrm{D}: \mathrm{P}=0.01)$ and AST (Group A: $\mathrm{P}=0.03$; Group B: $\mathrm{P}=0.002$; Group C: $\mathrm{P}=$ 0.0004; Group D: P = 0.0003) in Group A and Group B

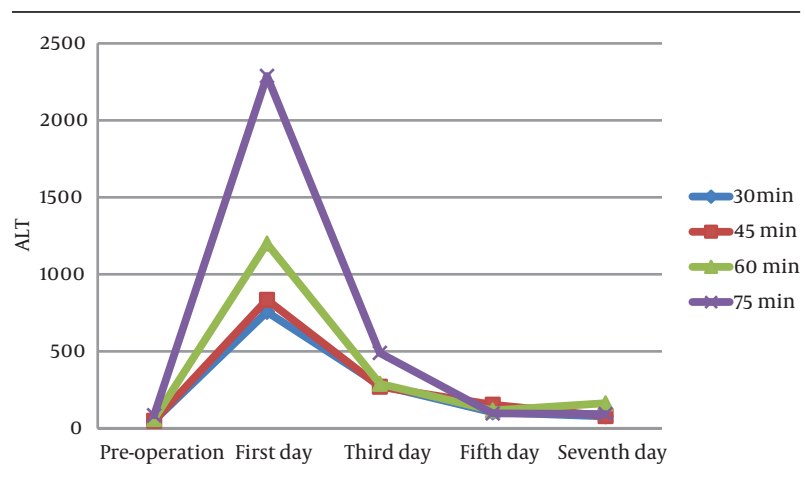

Figure 6. Alanine Aminotransferase Serum Activities Pre-Operatively to Seventh Day Post-Operative

significantly increased postoperatively and the maximum level was in the first day after operation. The postoperative maximum levels of AST and ALT in group C were significantly higher than those in groups A and B 
(Group A vs. Group C: P = 0.003; Group B vs. Group C: P = 0.0018 ) (Table 1). In Group D, the values were not significant, because the mortality rate in this group was higher than other groups and cases were not sufficient for comparison.

The mortality rate in groups A, B and C was $20 \%$, whereas $60 \%$ in group D.

\section{Discussion}

This study aimed to investigate safety time of portal clamping during Pringle maneuver (PM). Our clinical data showed that the safe upper limit of continues hepatic ischemia in normal liver can be extended to $45 \mathrm{~min}$ utes without major complications.

One of the major advances in liver surgery is prevention of intraoperative bleeding. One of the surgical methods applied to reduce blood loss during hepatectomy is inflow occlusion by clamping the portal triad (the Pringle maneuver). It significantly decreases bleeding during transection of the parenchyma and has little effect on systemic hemodynamics or liver function, particularly if applied intermittently (12-15). Although liver surgeons use different vascular clamping techniques, including PM, the optimal ischemia time has not yet been determined and remains controversial. Continuous clamping results in one prolonged period of ischemia followed by reperfusion, whereas during intermittent clamping multiple short periods of ischemia are induced, all followed by a reperfusion episode. The issue comes down to the question whether multiple short episodes of ischemia and reperfusion are more detrimental to the liver than one prolonged period of ischemia followed by final reperfusion.

This study aimed to investigate safe ischemic time of clamping during intermittent PM. In van Wagensveld et al. (16) study, INT ischemia was induced by clamping hepatic pedicle in eight successive periods of 12 minutes ischemia and 3 minutes of reperfusion. Intermittent hepatic vascular inflow occlusion during prolonged liver ischemia (120 minutes) in pigs resulted in less microcirculatory and hepatocellular injury, compared to continuous occlusion (17, 18).

In the clinical situation, intermittent clamping entails periods of 15 - 30 minutes of ischemia, followed by 5 - 15 minutes of reperfusion $(12,19)$. In our study, safe upper limit of continues hepatic ischemia in normal liver can be extended to 45 minutes without major complications. In all, an intermittent clamping permits increase of safe ischemic time up to 322 minutes in normal livers and 204 minutes in impaired livers and is the recommended method, especially in patients undergoing complex liver resections and patients with diseased livers (20).

In van den Broek study, no significant difference was found in aminotransferases level between IPM 15 minutes and IPM 30 minutes at any time point (20). In our study in Group A (30 minutes) and Group B (45 minutes), aminotransferases level significantly increased postop- eratively and the maximum level found in the first day after operation.

Kang study (21) found that repeated 30-minute ischemia interrupted by 5-minute reperfusion can be safely applied and is quite provocative and may have significant clinical impact. With IC, cumulative clamping periods of up to 322 minutes in normal liver and 204 minutes in cirrhotic livers have been reported $(20,21)$. Another study found that the two strategies were comparable as long as the ischemia time was less than or equal to 40 minutes. The markers of apoptosis were increased in the IP group if the ischemia time exceeded 40 minutes (22). Kim (22) study indicated that intermittent application of Pringle maneuver for 30 minutes each time can be complished effectively and safely for human hepatectomy when combined with protease inhibitor. van den Broek study showed that IPM with 15 or 30 minutes ischemic intervals induced similar hepatocellular injury measured by the sensitive marker L-FABP and suggested that IPM with 30 minutes ischemic intervals may be used (20). Ishizaki study showed that the safe upper limit of cumulative hepatic ischemia in normal liver can be extended to 325 minutes without major complications (23).

In conclusion, continues hepatic vascular inflow occlusion during liver ischemia (30 and 45 minutes) in rabbit resulted in no ischemic change. Increasing time of clamping to 30 minutes was safe in intermittent Pringle maneuver.

\section{Acknowledgments}

The authors wish to thank the members of animal laboratory of Isfahan University of Medical Sciences.

\section{Footnotes}

Authors' Contribution:Mohsen Kolahdoozan was the principal investigator of the study. Mehrdad Hosseinpour participated in drafting of the manuscript. Akbar Behdad was the attending professor of the thesis. Samin Behdad participated in drafting of the manuscript and contributed to the analysis. Mohammad Taghi Rezaei contributed to compiling data.

Funding/Support:This study was part of a thesis conducted in Isfahan University of Medical Sciences with the cooperation of the animal laboratoy of this university. This project was funded by Isfahan University of Medical Sciences.

\section{References}

1. Chouillard EK, Gumbs AA, Cherqui D. Vascular clamping in liver surgery: physiology, indications and techniques. Ann Surg Innov Res. 2010;4:2. doi:10.1186/1750-1164-4-2. [PubMed:20346153]

2. Lau WY, Lai EC, Lau SH. Methods of vascular control technique during liver resection: a comprehensive review. Hepatobiliary Pancreat Dis Int. 2010;9(5):473-81. [PubMed:20943455]

3. Kozar RA, Feliciano DV, Moore EE, Moore FA, Cocanour CS, West MA, et al. Western Trauma Association/critical decisions in trauma: operative management of adult blunt hepatic trau- 
ma. J Trauma. 2011;71(1):1-5. doi: 10.1097/TA.0b013e318220b192. [PubMed: 21818009]

4. Bernardo CG, Fuster J, Bombuy E, Sanchez S, Ferrer J, Loera MA, et al. Treatment of liver trauma: operative or conservative management. Gastroenterol research. 2010;3(1):9-18.

5. Tinkoff G, Esposito TJ, Reed J, Kilgo P, Fildes J, Pasquale M, et al. American Association for the Surgery of Trauma Organ Injury Scale I: spleen, liver, and kidney, validation based on the $\mathrm{Na}$ tional Trauma Data Bank. J Am Coll Surg. 2008;207(5):646-55. doi: 10.1016/j.jamcollsurg.2008.06.342. [PubMed:18954775]

6. Doklestic K, Stefanovic B, Gregoric P, Ivancevic N, Loncar Z, Jovanovic B, et al. Surgical management of AAST grades III-V hepatic trauma by Damage control surgery with perihepatic packing and Definitive hepatic repair-single centre experience. World J Emerg Surg. 2015;10:34. doi:10.1186/s13017-015-0031-8. [PubMed:26236391]

7. Latifi R, Khalaf H. Selective vascular isolation of the liver as part of initial damage control for grade 5 liver injuries: Shouldn't we use it more frequently? Int J Surg Case Rep. 2015;6C:292-5. doi: 10.1016/j.ijscr.2014.12.021. [PubMed: 25569195]

8. Benzoni E, Lorenzin D, Baccarani U, Adani GL, Favero A, Cojutti A, et al. Resective surgery for liver tumor: a multivariate analysis of causes and risk factors linked to postoperative complications. Hepatobiliary Pancreat Dis Int. 2006;5(4):526-33. [PubMed: 17085337]

9. Garcea G, Gescher A, Steward W, Dennison A, Berry D. Oxidative stress in humans following the Pringle manoeuvre. Hepatobiliary Pancreat Dis Int. 2006;5(2):210-4. [PubMed: 16698577]

10. Filos KS, Kirkilesis I, Spiliopoulou I, Scopa CD, Nikolopoulou V, Kouraklis G, et al. Bacterial translocation, endotoxaemia and apoptosis following Pringle manoeuvre in rats. Injury. 2004;35(1):35-43. [PubMed:14728953]

11. Grazi GL, Mazziotti A, Jovine E, Pierangeli F, Ercolani G, Gallucci A, et al. Total vascular exclusion of the liver during hepatic surgery. Selective use, extensive use, or abuse? Arch Surg. 1997;132(10):1104-9. [PubMed: 9336509]

12. Tralhao JG, Hoti E, Oliveiros B, Abrantes AM, Botelho MF, CastroSousa F. Intermittent pringle maneuver and hepatic function: perioperative monitoring by noninvasive ICG-clearance. World J Surg. 2009;33(12):2627-34. doi: 10.1007/s00268-009-0204-2. [PubMed: 19760319]

13. Xia F, Lau WY, Qian C, Ma K, Li X, Bie P. Continuous occlusion of hepatic artery proper for prevention of blood loss in partial hepatectomy for ruptured hepatocellular carcinoma: a casematched comparative study. Ann Surg Oncol. 2011;18(6):1638-43. doi:10.1245/s10434-010-1484-3. [PubMed: 21181280]

14. Belghiti J, Noun R, Malafosse R, Jagot P, Sauvanet A, Pierangeli F, et al. Continuous versus intermittent portal triad clamping for liver resection: a controlled study. Ann Surg. 1999;229(3):369-75. [PubMed:10077049]

15. Clavien PA, Yadav S, Sindram D, Bentley RC. Protective effects of ischemic preconditioning for liver resection performed under inflow occlusion in humans. Ann Surg. 2000;232(2):155-62. [PubMed:10903590]

16. van Wagensveld BA, van Gulik TM, Gelderblom HC, Scheepers J, et al. Continuous or intermittent vascular clamping during hemihepatectomy in pigs: hyaluronic acid kinetics in the assessment of early microvascular liver damage. European J of Surgery. 2000;166(3):255-261. doi: 10.1080/110241500750009375. [PubMed: 21975322]

17. Torzilli G, Procopio F, Donadon M, Del Fabbro D, Cimino M, Montorsi M. Safety of intermittent Pringle maneuver cumulative time exceeding 120 minutes in liver resection: a further step in favor of the "radical but conservative" policy. Ann Surg. 2012;255(2):270-80. doi:10.1097/SLA.ob013e318232b375. [PubMed: 21975322]

18. Yamaguchi N, Yokoyama Y, Ebata T, Igami T, Sugawara G, Asahara $\mathrm{T}$, et al. Intermittent Pringle maneuver is unlikely to induce bacterial translocation to the portal vein: a study using bacterium-specific ribosomal RNA-targeted reverse transcription-polymerase chain reaction. J Hepatobiliary Pancreat Sci. 2015;22(6):491-7. doi:10.1002/jhbp.239. [PubMed: 25782012]

19. Lei DX, Peng CH, Peng SY, Jiang XC, Wu YL, Shen HW. Safe upper limit of intermittent hepatic inflow occlusion for liver resection in cirrhotic rats. World J Gastroenterol. 2001;7(5):713-7. [PubMed: 11819861]

20. van den Broek MA, Bloemen JG, Dello SA, van de Poll MC, Olde Damink SW, Dejong $\mathrm{CH}$. Randomized controlled trial analyzing the effect of 15 or 30 min intermittent Pringle maneuver on hepatocellular damage during liver surgery. J Hepatol. 2011;55(2):33745. doi:10.1016/j.jhep.2010.11.024. [PubMed: 21147188]

21. Kang KJ, Jang JH, Lim TJ, Kang Y, Park KK, Lee IS, et al. Optimal cycle of intermittent portal triad clamping during liver resection in the murine liver. Liver Transpl. 2004;10(6):794-801. doi: 10.1002/lt.20177. [PubMed:15162475]

22. Kim YI, Fujita S, Hwang YJ, Chun JM, Song KE, Chun BY. Successful intermittent application of the Pringle maneuver for 30 minutes during human hepatectomy: a clinical randomized study with use of a protease inhibitor. Hepatogastroenterology. 2007;54(79):2055-60. [PubMed: 18251159]

23. Ishizaki Y, Yoshimoto J, Miwa K, Sugo H, Kawasaki S. Safety of prolonged intermittent pringle maneuver during hepatic resection. Arch Surg. 2006;141(7):649-53. doi: 10.1001/archsurg.141.7.649. [PubMed:16847234] 